\title{
Introducing our Tutorials
}

Nature Protocols launches a new Review-style article type, the Tutorial.

S ince its launch in 2006, Nature Protocols has been committed to publishing detailed protocols, penned by world-leading experts, for the best techniques. Our Protocols provide not only clear step-by-step procedures but also information about critical steps and troubleshooting guidance, as well as detailed introductions that explain further applications and limitations of the technique and compare the protocol with other available methods. Another section in our Protocols, of particular use to our readers, is the experimental design, in which the author presents carefully considered aspects of the technical details required to successfully implement a method.

In celebration of the importance of experimental design in method development and execution, we are launching a new Review-style article type, the Tutorial. Tutorials are devoted to the discussion of the thought process behind an experiment, and how to actually design an experiment using particular techniques. The purpose of these articles is to provide comprehensive guidelines and advice allowing researchers to adapt or apply a specific method to their own research, and permitting users to design their experiments from start to finish in a way that enables meaningful interpretation of the results. Unlike Protocols, which focus on a single method employed by the author's lab, Tutorials may discuss a greater range of methods used to investigate a specific biological question. We hope that these articles will answer practical questions that are likely to arise for researchers undertaking the methods, and provide information to guide their choices.

We are pleased to highlight in this issue our first Tutorial, by Holger Heyn and colleagues, describing the experimental design considerations for single-cell transcriptomics approaches. Here the authors provide a framework for designing single-cell RNA sequencing experiments and discuss sample-preparation aspects, the choice of sequencing methods, and data processing and analysis approaches, which they hope will provide a complete guide to support users in designing their own experiments all the way from sample preparation to data interpretation.

We pride ourselves on publishing protocols that include detailed information about methods that cannot be found elsewhere, and we hope that these Tutorials will add the same value, providing necessary information for researchers who want to learn a new technique or adapt existing methods to address their own scientific questions.

We hope you enjoy reading our Tutorials, and if you have any comments or suggestions, we would love to hear from you.

Published online: 16 November 2018 https://doi.org/10.1038/s41596-018-0078-6 